\title{
Integration of functional neuroimaging in CyberKnife radiosurgery: feasibility and dosimetric results
}

\author{
Alfredo Conti, M.D., Ph.D., ${ }^{1}$ Antonio Pontoriero, M.D., ${ }^{2}$ Giuseppe K. Ricciardi, M.D., ${ }^{3}$ \\ Francesca Granata, M.D. ${ }^{4}{ }^{4}$ Sergio Vinci, M.D., ${ }^{4}$ Filippo F. Angileri, M.D., Ph.D., 1 \\ Stefano Pergolizzi, M.D., ${ }^{3}$ Concetta Alafaci, M.D.,${ }^{1}$ Vincenzo Rizzo, M.D., 5 \\ Angelo Quartarone, M.D., 5 Antonino Germanò, M.D., ${ }^{1}$ Roberto Israel Foroni, Ph.D., 6 \\ Costantino De Renzis, M.D., and Francesco Tomasello, M.D. ${ }^{2}$
}

Departments of ${ }^{I}$ Neurosurgery and ${ }^{2}$ Radiation Oncology, CyberKnife Center, University of Messina; Departments of ${ }^{4}$ Neuroradiology and ${ }^{5}$ Neurology, University of Messina; and Departments of ${ }^{3}$ Neuroradiology and ${ }^{6}$ Stereotaxis, University of Verona, Italy

Object. The integration of state-of-the-art neuroimaging into treatment planning may increase the therapeutic potential of stereotactic radiosurgery. Functional neuroimaging, including functional MRI, navigated brain stimulation, and diffusion tensor imaging-based tractography, may guide the orientation of radiation beams to decrease the dose to critical cortical and subcortical areas. The authors describe their method of integrating functional neuroimaging technology into radiosurgical treatment planning using the CyberKnife radiosurgery system.

Methods. The records of all patients who had undergone radiosurgery for brain lesions at the CyberKnife Center of the University of Messina, Italy, between July 2010 and July 2012 were analyzed. Among patients with brain lesions in critical areas, treatment planning with the integration of functional neuroimaging was performed in 25 patients. Morphological and functional imaging data sets were coregistered using the Multiplan dedicated treatment planning system. Treatment planning was initially based on morphological data; radiation dose distribution was then corrected in relation to the functionally relevant cortical and subcortical areas. The change in radiation dose distribution was then calculated.

Results. The data sets could be easily and reliably integrated into the Cyberknife treatment planning. Using an inverse planning algorithm, the authors achieved an average $17 \%$ reduction in the radiation dose to functional areas. Further gain in terms of dose sparing compromised other important treatment parameters, including target coverage, conformality index, and number of monitor units. No neurological deficit due to radiation was recorded at the shortterm follow-up.

Conclusions. Radiosurgery treatments rely on the quality of neuroimaging. The integration of functional data allows a reduction in radiation doses to functional organs at risk, including critical cortical areas, subcortical tracts, and vascular structures. The relative simplicity of integrating functional neuroimaging into radiosurgery warrants further research to implement, standardize, and identify the limits of this procedure.

(http://thejns.org/doi/abs/10.3171/2013.2.FOCUS12414)

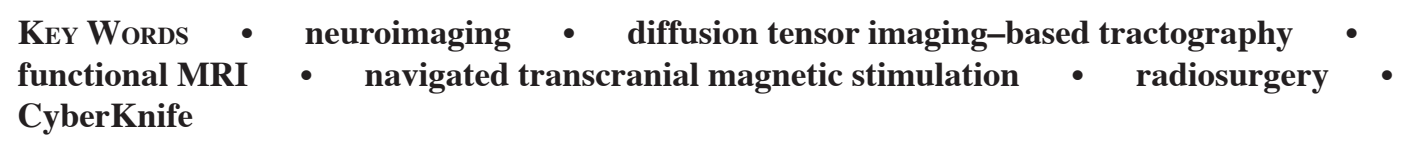

$\mathrm{S}$ TEREOTACTIC radiosurgery has become a mainstream neurosurgical procedure. It is routinely used as either a definitive or an adjuvant treatment modality in the fields of neurooncology, cerebrovascular disease, and functional neurosurgery. Ongoing research is fo-

\footnotetext{
Abbreviations used in this paper: $\mathrm{AVM}=$ arteriovenous malformation; DTI = diffusion tensor imaging; EMG = electromyography; fMRI = functional MRI; NBS = navigated brain stimulation; TMS = transcranial magnetic stimulation.
}

cused on new energy sources and improvements in radiation delivery precision. Readily available opportunities to improve stereotactic radiosurgery can be obtained via the integration of sophisticated neuroimaging techniques into treatment planning.

State-of-the-art functional neuroimaging may provide a valuable complement to conventional treatments by guiding the orientation of radiation beams to decrease the dose to critical cortical and subcortical areas. Functional MRI can be used to define the location of func- 


\section{A. Conti et al.}

tionally eloquent cortices, whereas DTI-based tractography displays the distribution and abnormalities of white matter tracts. This information can be integrated into the radiosurgical treatment plan to improve sparing of critical structures. ${ }^{4}$ We have recently integrated NBS into CyberKnife treatments. Navigated brain stimulation is a technique for mapping the motor cortex by applying TMS to brain areas. ${ }^{11-14}$ In NBS, conventional TMS is combined with sophisticated neuronavigation software that allows the navigation of each single "stimulated" point in the cortex. The electromagnetic stimulus delivered by the TMS coil overcomes the activation threshold of the underlying motor neurons so that the impulse can propagate to the corticospinal tract determining a muscle contraction. Muscle responses to the electric field (motor evoked potential) are recorded using EMG channels.

The aim in this study was to analyze the technical possibility of integrating state-of-the-art functional neuroimaging into CyberKnife radiosurgery treatment plans. We also report the average rate of critical structure sparing achieved and outline cases in which integrated neuroimaging allowed the creation of treatment plans that differed markedly from what would have been possible with $\mathrm{CT}$ and/or MRI.

\section{Methods}

We retrospectively reviewed the database of patients who had undergone stereotactic radiosurgery to the brain via a robotic radiosurgery system at the CyberKnife Center of the University of Messina in Italy. Between July 2010 and July 2012, 25 patients with brain lesions in critical areas underwent functional neuroimaging. All patients underwent morphological MRI and CT for CyberKnife radiosurgery treatment. Image acquisitions, processing of the imaging data, and CyberKnife treatment planning have been described elsewhere. ${ }^{1,2}$ The modalities of acquisition and integration of functional imaging are described in detail.

\section{Diffusion Tensor Imaging and Fiber Tracking}

Using a 3-T MRI unit, we acquired a high-resolution T1-weighted isotropic volumetric data set with a 3D magnetization-prepared rapid acquisition gradient-echo (3D-MPRAGE) sequence with a $1 \times 1 \times 1-\mathrm{mm}$ voxel size. Diffusion tensor imaging was performed during the same session. Patients were imaged with a parallel imaging technique (integrated parallel acquisition technique [iPAT] generalized autocalibrating partially parallel acquisition [GRAPPA] implementation with an acceleration factor of 2) and a 4-channel coil. A diffusion-weighted echo-planar sequence and a diffusion-weighted singleshot spin-echo echo-planar sequence were acquired with an isotropic voxel of $2.3 \times 2.3 \times 2.3 \mathrm{~mm}$.

Diffusion tensor images were transferred to a personal computer, converted to ANALYZE format, and initially corrected for the effects of eddy current-induced distortion using FSL modules. After returning them to the DICOM format, the images were processed with Diffusion Toolkit software (Martinos Center for Biomedical Imaging, Massachusetts General Hospital) to calculate voxel-based fractional anisotropy maps. Fiber tracking was performed with the same software using the interpolated streamline propagation algorithm.

All 3D tract reconstructions were obtained using TrackVis software (Martinos Center for Biomedical Imaging; Fig. 1A-C).

After each tracking, fractional anisotropy volumes were resliced in the axial, coronal, and sagittal planes and displayed in conjunction with tractography results to allow neuroanatomical location of the tract reconstructions (Fig. 1D). The higher-resolution T1-weighted images were used to resolve occasional ambiguities when identifying seeding regions of interest in the lower-resolution fractional anisotropy or non-diffusion-weighted images.

\section{Blood Oxygen Level-Dependent fMRI}

Functional MRI data were acquired on the 3-T MR scanner equipped with echo-planar imaging capability and a standard quadrature head coil. Images were obtained using a standard gradient-echo echo-planar sequence. A T1-weighted anatomical image was also acquired for each patient. Functional data were analyzed using BrainVoyager QX (Version 1.9, Brain Innovation). Preprocessing of fMRI data included 3D motion correction, slice scan time correction (since interpolation), linear trend removal by temporal high-pass filtering ( 3 cycles per time course), and transformation into the Talairach coordinate space.

Activated voxels were identified with a single-subject general linear model approach for time series data. To account for the hemodynamic delay, the boxcar waveform representing the rest and task conditions was convolved with an empirical hemodynamic response function. Brain activation was detected by comparing blood oxygen level-dependent (BOLD) signal intensities in fMR images acquired during the stimulation task (TASK) and in patients at rest (REST). Individual statistical maps were thresholded at pb.05 and corrected for multiple comparisons (Bonferroni). Thresholded statistical functional maps were then superimposed on the respective structural scans to localize significantly activated areas.

A Macintosh laptop computer running PsyScope software, a graphical user interface software program that runs psychological experiments, was used to present the visual stimuli for each task. Before performing the tasks, all patients underwent a brief training session with PsyScope. Patients were provided with written and verbal instructions and could practice the tasks while the investigators monitored their performance. Visual stimuli were projected onto a screen located at the end of the MR scanner table by using the Presentation software package (www.neurobs.com), and the patient viewed the screen via a mirror on top of the head coil. Auditory stimuli were delivered to the patient through MRI-compatible headphones (Resonance Technologies, Inc.) using the same software package. Movement of the patient's head was restrained with a moldable air bag (Vac Fix immobilization system, Bionix Radiation Therapy). The following 6 language tasks were used, because they are believed to test a full range of language functions thought to involve both frontal and temporoparietal language areas: con- 


\section{Functional neuroimaging in CyberKnife radiosurgery}
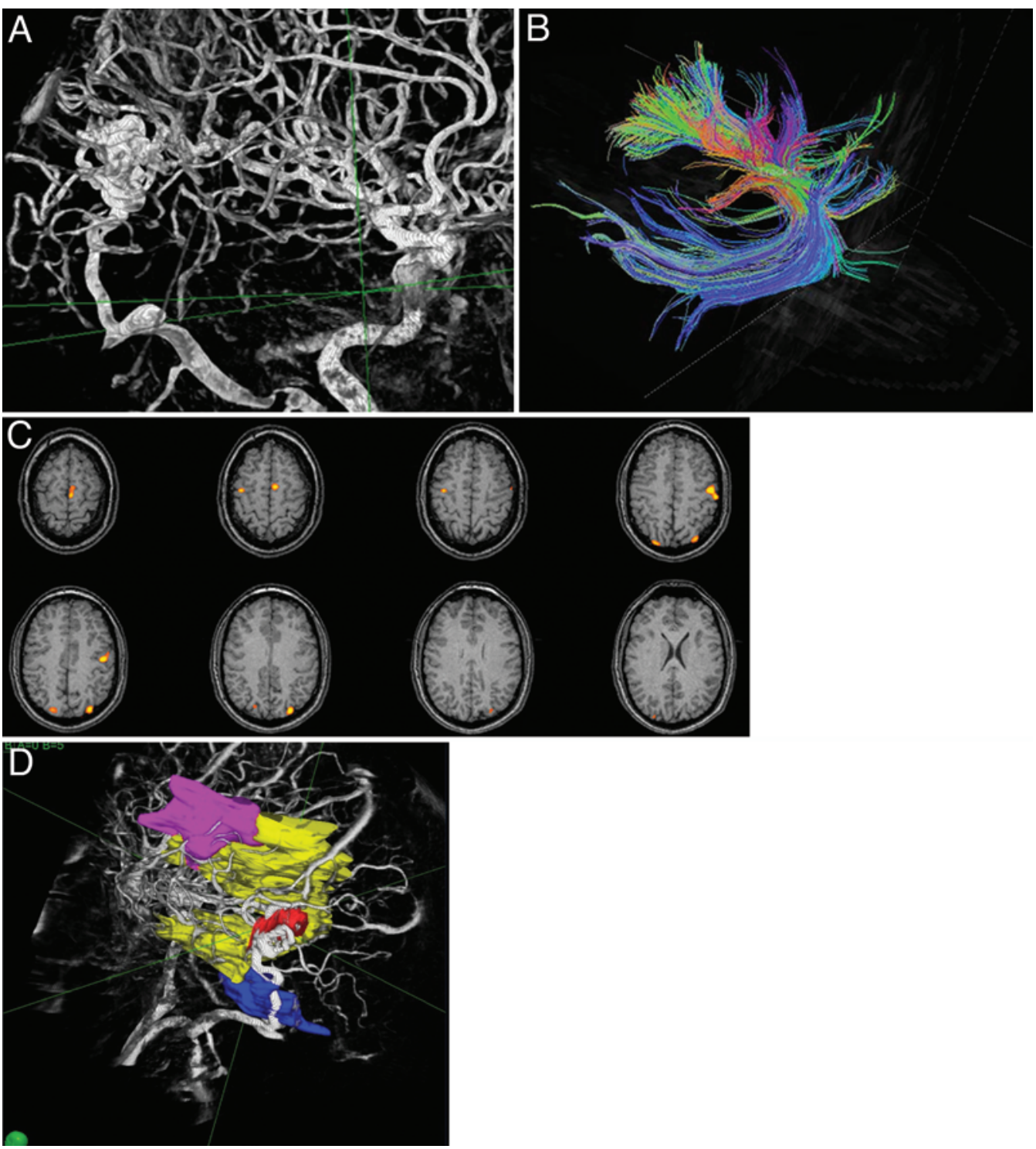

FIG. 1. Sophisticated imaging data sets were integrated into the radiosurgical treatment of cerebral AVMs. A 3D rotational angiogram (A) showing a left temporal AVM. A DTI tractography image (B) demonstrating the arcuate fasciculus. Functional MR images (C) obtained in the same patient, showing cortical areas activated by verbal tasks. Functional and tractographic MR images were coregistered and rendered in 3D. Functional neuroimaging data were incorporated into 3D rotational angiography data (D) for AVM treatment. The target lesion (red) is visible, as are the left-sided arcuate fasciculus (yellow), Broca area (purple), and Wernicke area (blue).

frontation naming, verb generation, visually presented sentence comprehension, orally presented sentence comprehension, semantic decision making, and story listening. The motor system was explored with finger tapping, ankle plantar flexion, and tongue tapping.

\section{Navigated Brain Stimulation}

Mapping of the motor cortex using navigated TMS was performed using an NBS system (NBS System 4, Nexstim, Ltd.). Transcranial magnetic stimulation was performed using a figure- 8 coil applied perpendicular to the sagittal line of the patient's head, with an inclination of $45^{\circ}$ and a posterior-to-anterior flow of direct current. In the NBS system, TMS is associated with neuronavi- gated tracking of the stimulation points in the cortex. The procedure was performed using the following 6 steps. 1) The patient's brain volumetric MRI data were uploaded into the NBS system to elaborate a 3D reconstruction. A dedicated tool of the NBS system allowed layer-by-layer peeling of the skin, bone, and meninges to obtain a 3D visualization of the cortical surface. 2) The electrodes for recording electromyographic activity were placed on the surface of the muscles to be activated. The amplitude and latency of evoked potentials from 6 EMG channels were automatically calculated and recorded. 3) The patient wore special eye frames for the head tracking. The operator then tracked the patient's head with a pointer. The NBS system aligned the points virtually drawn on the patient's head with their corresponding coordinates 
on the 3D MR rendering. 4) Before NBS mapping, the resting motor threshold of each patient was determined. The resting motor threshold is the minimal intensity of stimulation that can elicit at least 5 of 10 motor evoked potentials, with an amplitude of $50 \mathrm{mV}$. The cortex of each patient was activated with a stimulation intensity 20\%-30\% higher than the resting motor threshold. 5) A cortical somatotopic map was generated by stimulating different parts of the cortex and identifying those areas in which the motor response was more intense. 6) The whole data set, including the volumetric MRI data and the coregistered functional map, was exported and uploaded into the operative CyberKnife system.

\section{Results}

In 25 patients with lesions in critical areas, functional neuroimaging modalities were integrated into the radiosurgical treatment planning system. Functional data sets were coregistered with a morphological T1-weighted MR sequence using the dedicated treatment planning system Multiplan (Accuray, Inc.). Functional data included fMRI and DTI-based tractography data in 10 patients with AVMs and 3 patients with brain metastases and NBSbased identification of the motor cortex in 12 patients with brain tumors.

With regard to the integration procedure, raw fMR images had to be coregistered with an anatomical, volumetric MR image for each patient by using an offline procedure. Similarly, the color-coded fractional anisotropy maps were fused with the anatomical MR images using the 3D Slicer software package (www.slicer.org;
Fig. 1A-C). The resulting data set was a 3D T1-weighted MR sequence integrating functional information that was used for radiosurgery planning. Navigated brain stimulation images consisted of 2 data sets: the morphological imaging data and the functional data. The latter consisted of a volumetric set of images with the same spatial information as the morphological data set from which they were extracted. Accordingly, the integration of the NBS data set did not generate spatial errors.

The functionally relevant structures of the brain and the fiber tracts situated near the target were delineated using the fused activation maps and tractography images. The motor cortex and pyramidal tract, the verbal cortices, and the arcuate fasciculus connecting the motor and sensitive verbal cortices were identified and used as functionally critical volumes (Fig. 2). In specific situations, such as AVMs located in critical areas, we combined multiple imaging modalities such as 3D rotational angiography, DTI tractography, and fMRI (Fig. 1D).

For radiosurgical treatment planning, the maximum allowed dose values were assigned to the target and several critical volumes, and a strict order of clinical goals was optimized according to their priority. Results showed that functionally relevant structures and fiber tracts could receive radiation doses as great as those prescribed to the target when the treatment plan was not optimized for functional data. The integration of fMRI data allowed us to reduce doses to critical brain areas by $200-500$ cGy (average $350 \mathrm{cGy}$ ) when the maximal dose to the target ranged from 2500 to $2750 \mathrm{cGy}$ and the dose prescribed to the target periphery averaged $2000 \mathrm{cGy}$. Accordingly, we achieved up to a $25 \%$ reduction (average 17\%) in the

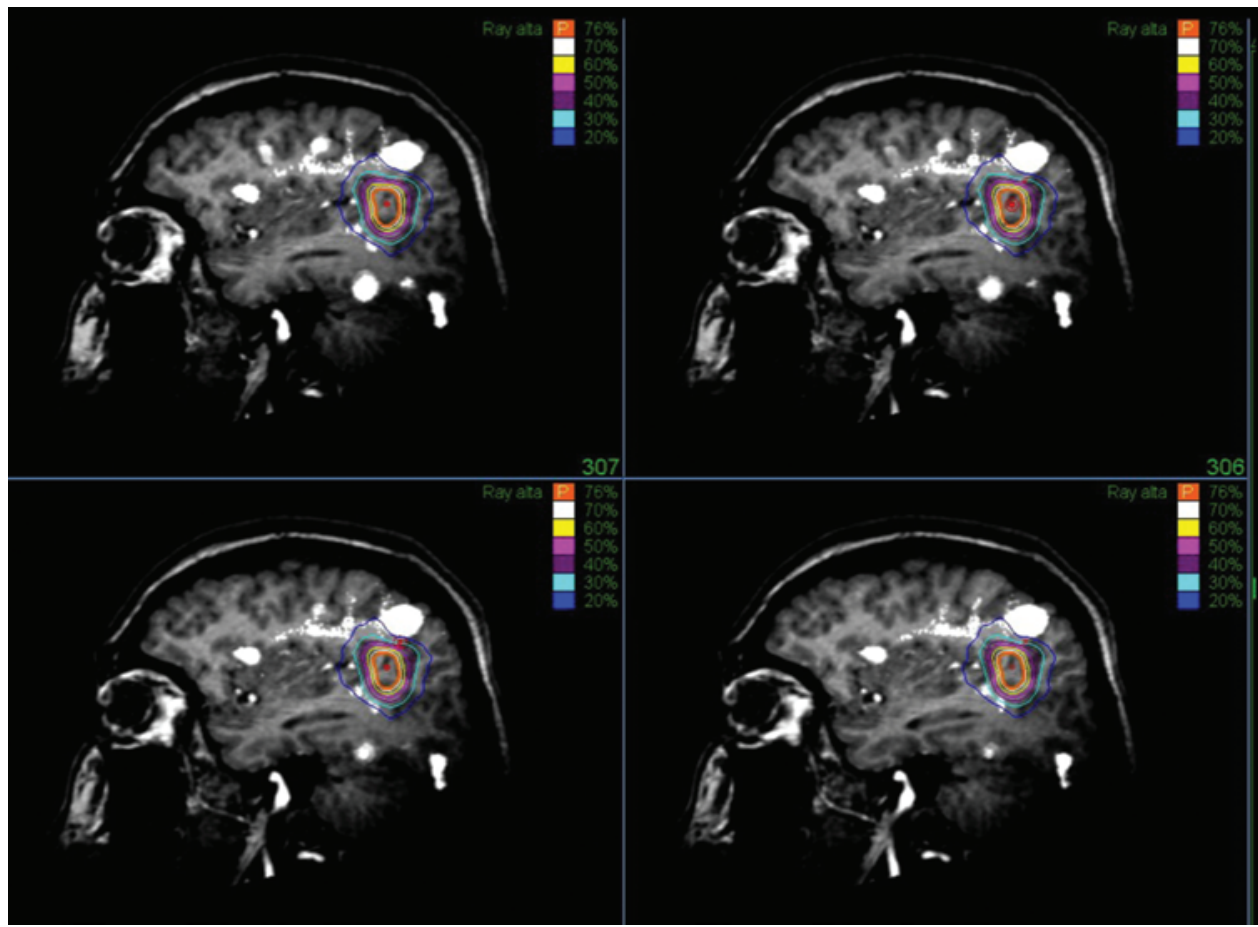

FIG. 2. Voxels generated with fMRI (solid white areas indicate cortices activated by language tasks) and DTI tractography (dotted white areas represent the arcuate fasciculus) were spatially localized and reported in a morphological T1-weighted MRI volume (sequential sagittal views). Radiation dose values were assigned to the target and several critical volumes, and a strict order of clinical goals was optimized according to their priority. 


\section{Functional neuroimaging in CyberKnife radiosurgery}

radiation dose after treatment plan optimization for critical functional volumes (Fig. 3).

Upon including critical cortical and subcortical structures in the optimization process, we noted that the size and/or number of collimators had to be reduced in most cases. The number of monitor units and beams increased if the dose constraints to functional critical volumes were more stringent. Attempting further gains in terms of dose sparing, when a lesion was very close to functionally relevant structures, led to an increase in the integral dose, which was calculated as the mean dose to all target and critical structures multiplied by the volume of all soft tissues, and the time of treatment. Alternatively, the planning resulted in an excessive loss of coverage of the target lesion.

We also calculated the volume of functional motor cortex when it was defined using fMRI or NBS. The cortical surface considered functionally relevant was $30 \%$ smaller when defined with NBS as compared with its definition via fMRI. Because of the smaller volumes, functional data obtained with NBS allowed us to achieve greater dose reductions to cortical functional areas that averaged $25 \%$.

With regard to clinical results, 10 patients with
AVMs had a mean follow-up of 17 months; 12 with malignant brain tumors, 9 months; and 3 with meningiomas, 8 months. No patient harbored neurological deficits attributable to the irradiation of critical structures at the last available follow-up.

\section{Discussion}

The use of fMRI and DTI tractography is gaining interest in the neurosurgical literature. ${ }^{3,5}$ At many centers, these sophisticated neuroimaging modalities have entered daily neurosurgery practice and have been used to plan image-guided resections of lesions located in critical areas. Despite a limited number of published studies on this topic, the value of such functional information in radiosurgical planning for lesions in or around eloquent cortex and subcortical white matter has been recognized. ${ }^{6-10,15}$ In fact, radiosurgery uses ablative doses of radiation but without the possibility of direct brain mapping and monitoring. Therefore, pretreatment mapping remains the only means of integrating functional information into stereotactic radiosurgery.

We integrated multiple imaging modalities into our CyberKnife treatments, including fMRI, DTI tractogra-

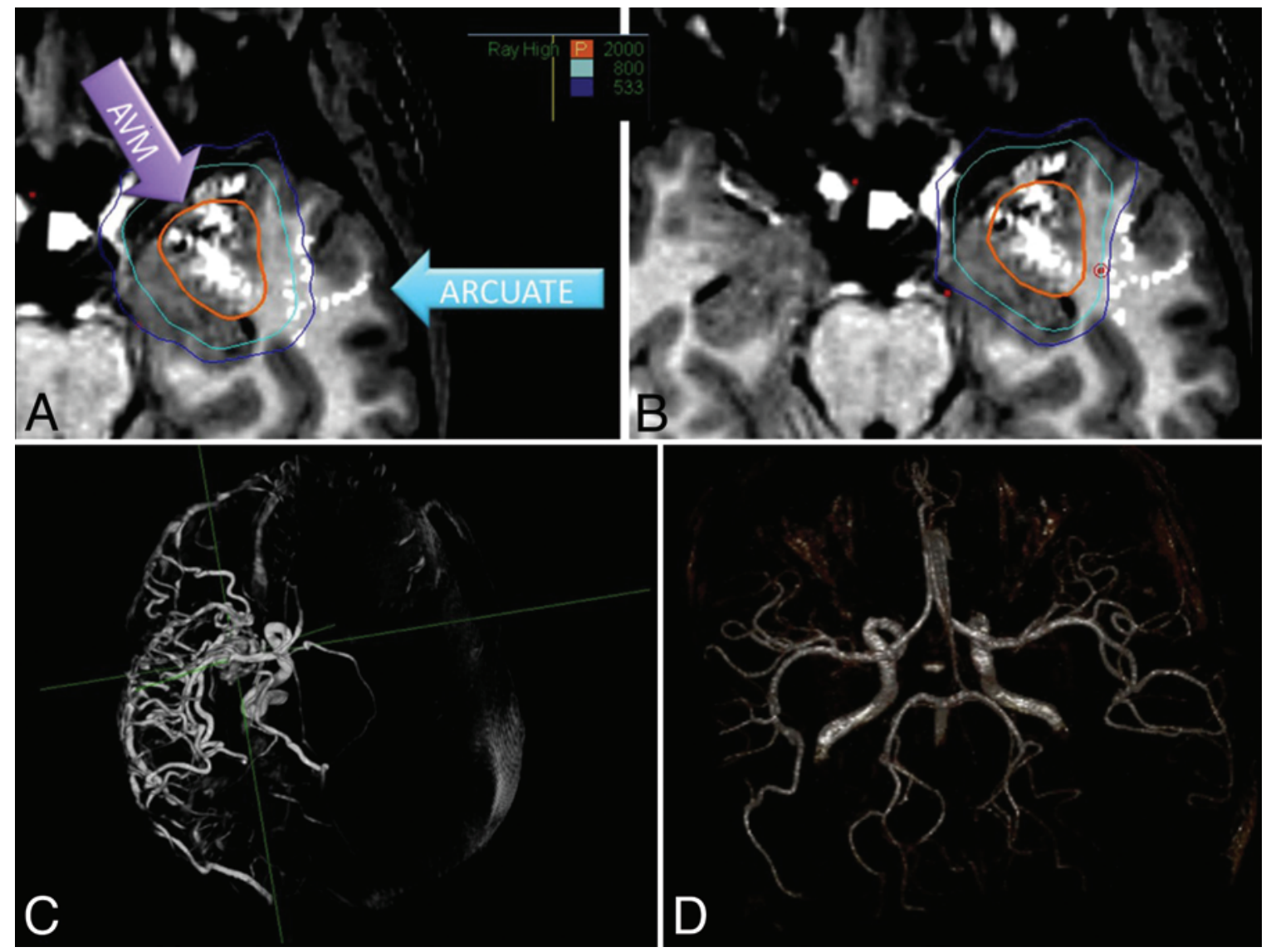

FIG. 3. Diffusion tensor imaging-based tractography data could be displayed on a morphological 3-T MRI sequence. Dotted white areas represent the arcuate fasciculus adjacent to a left temporal AVM. Cobalt blue, aqua, and orange lines represent isodose lines. Using an inverse planning algorithm, it was possible to reduce radiation to the arcuate fasciculus as shown by isodose distributions before (A) and after (B) optimization; however, this required changing of important treatment parameters, including the size of collimators and prescription isodoses. The left temporal AVM (C, 3D rotational angiograph) disappeared 18 months later (D, MR angiograph) without complications. 
phy, and, more recently, NBS. Functional structures of the brain and fiber tracts situated near the target were delineated using fused activation maps and tractography images. In specific situations, such as AVMs located in critical areas, we could combine data from multiple imaging modalities including 3D rotational angiography, DTI tractography, and fMRI (Fig. 1). During radiosurgery planning, the maximum allowed doses were assigned to the target and critical volumes, and a strict order of clinical goals was optimized according to their priority by using an inverse planning algorithm. The doses delivered to critical regions could be reduced by up to $25 \%$ of their initial values after the integration of functional data. $\mathrm{Re}-$ markably, the identification of critical structures allowed us to keep these structures outside the prescription isodose line, which was as high as 2200 cGy for AVMs or brain metastases.

Upon including critical cortical and subcortical structures in the optimization process, we noted that the size and/or number of collimators had to be reduced in most cases, so that the number of monitor units and beams increased along with the treatment time. Furthermore, this led to an increase in the integral dose, defined as the mean dose to all target and critical structures multiplied by the volume of all soft tissues.

Accordingly, the optimization of the treatment plan that can be achieved with radiosurgery systems seems to have definite limits. For the motor system, the dose reduc- tion that can be achieved with the integration of functional neuroimaging might be sufficient to grant a low probability of radiation-induced complications. Maruyama and colleagues ${ }^{6}$ indicated a dose limit of 23 Gy for the pyramidal tract to maintain a $<5 \%$ risk of complications. The treatment plan can be easily optimized to this limit, even in most complex cases (Fig. 4). The radiation doses tolerated by the arcuate fasciculus or the optic pathway seem to be much lower, on the order of $10 \mathrm{~Gy}$, according to preliminary observations made by Maruyama and colleagues. ${ }^{8,9}$ This means that, for lesions lying close to these critical structures, it is not always possible to stay within the suggested limits while delivering biologically effective doses.

In this regard, it is particularly important to avoid the overestimation of critical volumes. Navigated brain stimulation offers a number of advantages in defining critical volumes. The spatial resolution of NBS is actually extremely high, since we can record the differences in cortical responses within a few millimeters. Furthermore, NBS does not passively record brain activity during voluntary patient movements relying on cerebral blood flow or metabolism, which could be altered in the presence of brain tumors and vascular malformations, but instead detects a direct electromyographic response evoked by TMS (Fig. 5).

Moreover, fMRI displays all cortical areas involved in a specific cognitive activity. For complex functions,
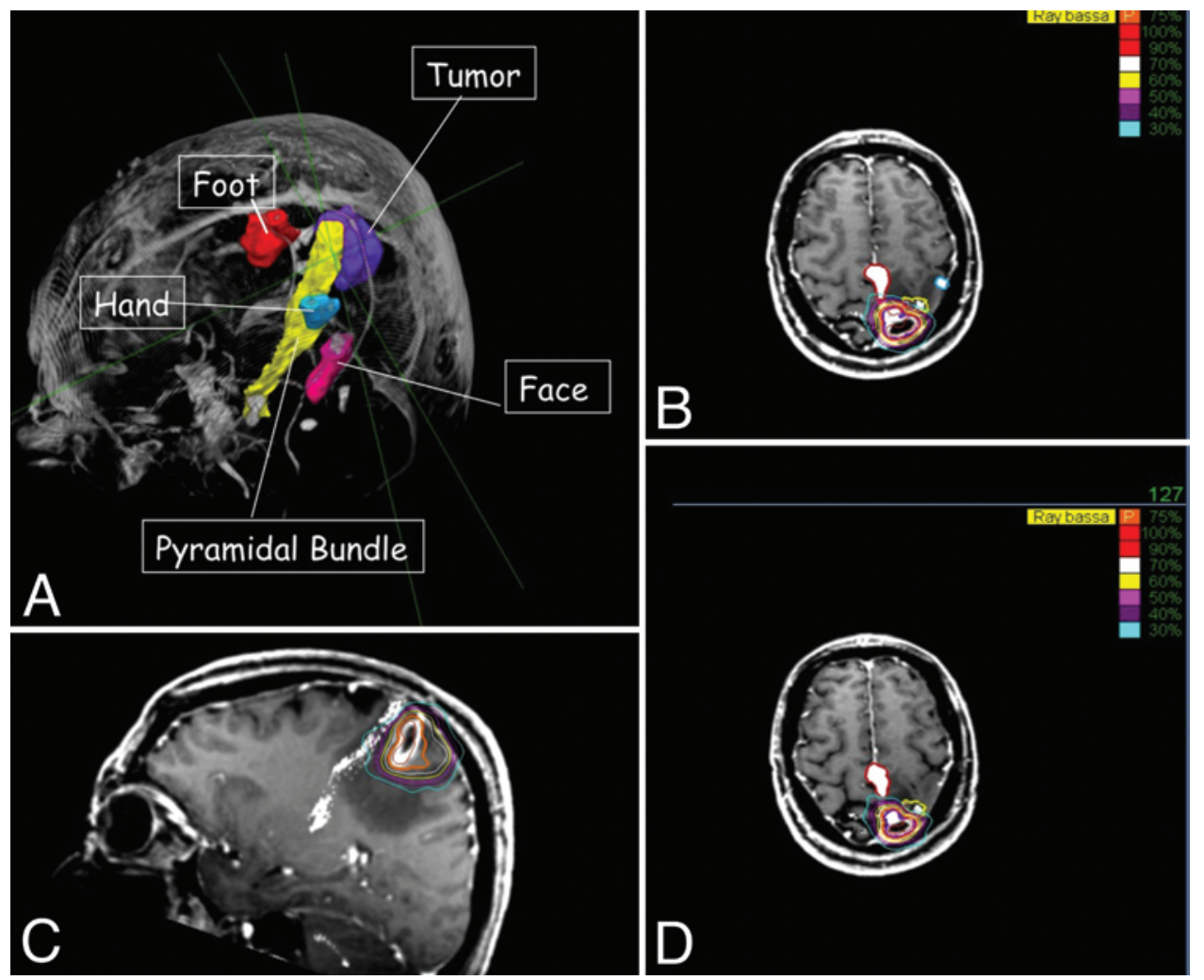

FIG. 4. Three-dimensional rendering (A) of combined morphological MRI, DTI tractography, and fMRI, showing a postrolandic tumor (purple). Combining different imaging modalities, it is possible to identify a draining vein coursing over the tumor; the functional motor areas corresponding to the right foot, hand, and face; and the pyramidal bundle. Voxels generated with fMRI and DTI tractography were spatially localized and incorporated into a morphological T1-weighted MRI volume, sagittal (C) and axial (B and D) views. Radiation dose values were assigned to the target and several critical volumes. 


\section{Functional neuroimaging in CyberKnife radiosurgery}

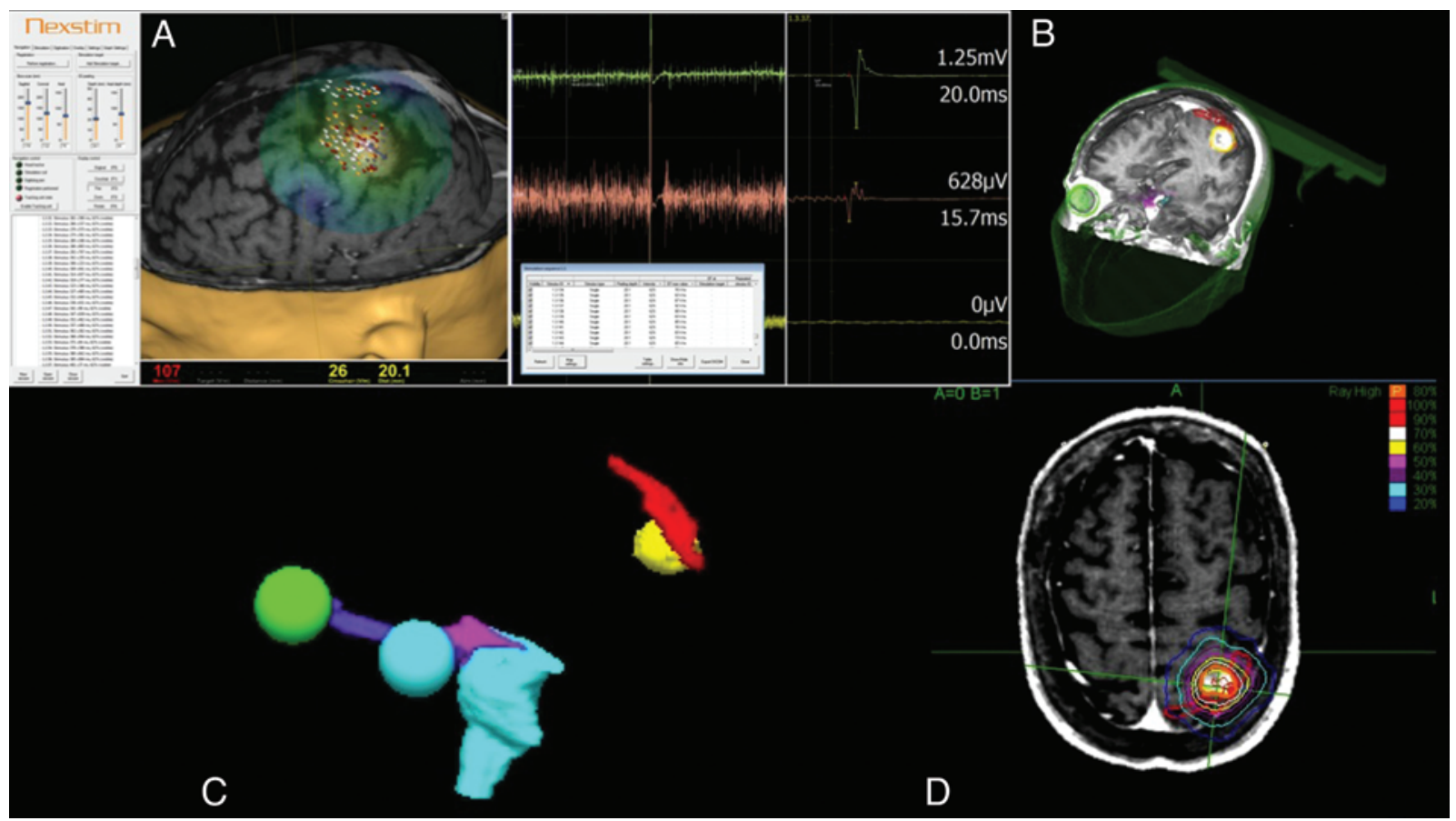

FIG. 5. The NBS system determines the actual location of the stimulating electric field in the cortex. In moving the TMS coil over the patient's head, it is possible to see in real time the stimulation location, strength, and direction in a 3D intracranial rendering (A). Volumetric visualization of prescription isodose and functional motor area (B). During mapping, the areas in the cortex with maximal EMG responses are automatically highlighted. The functional map can be exported and integrated into the CyberKnife treatment planning system to be rendered in 3D (C) and used for planning (D).

many areas are simultaneously activated by the task performed by a patient and are visualized together on fMRI. Navigated brain stimulation can be used in the repetitive modality, by which a train of impulses may inhibit the activity of the stimulated cortical surface. The combination of complex fMRI maps and navigated repetitive TMS may disclose the hierarchy of the different areas and identify primary functional areas that should be more carefully preserved during surgical or radiosurgical procedures.

\section{Conclusions}

The integration of functional neuroimaging into radiosurgery represents an independent and substantially unexplored opportunity to improve this treatment option. Demonstrating and quantifying the benefit for patients, in terms of safety and efficacy, is difficult to establish. The problem of the spatial accuracy of additional data and the spatial distortion induced by the coregistration process still need to be definitively addressed. Despite these limitations, the potential advantage in dealing with lesions located in critical brain areas and the relative simplicity of integrating functional neuroimaging into radiosurgery warrant further research.

\section{Disclosure}

This study was funded in part by Grant No. PRIN 2010ZESJWN from the Italian Ministry for University and Research.

Author contributions to the study and manuscript preparation include the following. Conception and design: Conti, Angileri, Pergolizzi, Tomasello. Acquisition of data: Conti, Pontoriero, Ric- ciardi, Granata, Vinci, Rizzo, Quartarone, Foroni. Analysis and interpretation of data: Conti, Pontoriero, Ricciardi, Granata, Vinci, Quartarone, Germanò. Drafting the article: Conti. Critically revising the article: Angileri, Pergolizzi, Alafaci, De Renzis. Approved the final version of the manuscript on behalf of all authors: Conti. Study supervision: Alafaci, Germanò, De Renzis, Tomasello.

\section{Acknowledgment}

The authors are grateful to Dr. Dave Schaal.

\section{References}

1. Conti A, Pontoriero A, Faragò G, Midili F, Siragusa C, Granata F, et al: Integration of three-dimensional rotational angiography in radiosurgical treatment planning of cerebral arteriovenous malformations. Int J Radiat Oncol Biol Phys 81:e29-e37, 2011

2. Conti A, Pontoriero A, Salamone I, Siragusa C, Midili F, La Torre D, et al: Protecting venous structures during radiosurgery for parasagittal meningiomas. Neurosurg Focus 27(5): E11, 2009

3. Jbabdi S, Mandonnet E, Duffau H, Capelle L, Swanson KR, Pélégrini-Issac M, et al: Simulation of anisotropic growth of low-grade gliomas using diffusion tensor imaging. Magn Reson Med 54:616-624, 2005

4. Krishnan AP, Asher IM, Davis D, Okunieff P, O'Dell WG: Evidence that MR diffusion tensor imaging (tractography) predicts the natural history of regional progression in patients irradiated conformally for primary brain tumors. Int J Radiat Oncol Biol Phys 71:1553-1562, 2008

5. Leclercq D, Duffau H, Delmaire C, Capelle L, Gatignol P, Ducros M, et al: Comparison of diffusion tensor imaging tractography of language tracts and intraoperative subcortical stimulations. Clinical article. J Neurosurg 112:503-511, 2010 


\section{A. Conti et al.}

6. Maruyama K, Kamada K, Ota T, Koga T, Itoh D, Ino K, et al: Tolerance of pyramidal tract to gamma knife radiosurgery based on diffusion-tensor tractography. Int J Radiat Oncol Biol Phys 70:1330-1335, 2008

7. Maruyama K, Kamada K, Shin M, Itoh D, Aoki S, Masutani $\mathrm{Y}$, et al: Integration of three-dimensional corticospinal tractography into treatment planning for gamma knife surgery. $\mathbf{J}$ Neurosurg 102:673-677, 2005

8. Maruyama K, Kamada K, Shin M, Itoh D, Masutani Y, Ino K, et al: Optic radiation tractography integrated into simulated treatment planning for Gamma Knife surgery. J Neurosurg 107:721-726, 2007

9. Maruyama K, Koga T, Kamada K, Ota T, Itoh D, Ino K, et al: Arcuate fasciculus tractography integrated into Gamma Knife surgery. Clinical article. J Neurosurg 111:520-526, 2009

10. Pantelis E, Papadakis N, Verigos K, Stathochristopoulou I, Antypas C, Lekas L, et al: Integration of functional MRI and white matter tractography in stereotactic radiosurgery clinical practice. Int J Radiat Oncol Biol Phys 78:257-267, 2010

11. Picht T, Schmidt S, Brandt S, Frey D, Hannula H, Neuvonen T, et al: Preoperative functional mapping for rolandic brain tumor surgery: comparison of navigated transcranial magnetic stimulation to direct cortical stimulation. Neurosurgery 69: 581-588, 2011

12. Picht T, Schmidt S, Woitzik J, Suess O: Navigated brain stim- ulation for preoperative cortical mapping in paretic patients: case report of a hemiplegic patient. Neurosurgery 68:E1475E1480, 2011

13. Picht T, Schulz J, Hanna M, Schmidt S, Suess O, Vajkoczy P: Assessment of the influence of navigated transcranial magnetic stimulation on surgical planning for tumors in or near the motor cortex. Neurosurgery 70:1248-1257, 2012

14. Sollmann N, Picht T, Makela JP, Meyer B, Ringel F, Krieg SM: Navigated transcranial magnetic stimulation for preoperative language mapping in a patient with a left frontoopercular glioblastoma. Case report. J Neurosurg 118:175-179, 2013

15. Stancanello J, Cavedon C, Francescon P, Causin F, Avanzo M, Colombo F, et al: BOLD fMRI integration into radiosurgery treatment planning of cerebral vascular malformations. Med Phys 34:1176-1184, 2007

Manuscript submitted December 15, 2012.

Accepted February 1, 2013.

Please include this information when citing this paper: DOI: 10.3171/2013.2.FOCUS12414.

Address correspondence to: Alfredo Conti, M.D., Ph.D., Department of Neurosurgery, CyberKnife Center, Via Consolare Valeria, 1, Messina 98125, Italy. email: alfredo.conti@unime.it. 